\title{
Algorithms and software for diffractive optics
}

Frank Wyrowski, Harald Aagedal, Thomas Beth, Michael Schmid

Frank Wyrowski, Harald Aagedal, Thomas Beth, Michael Schmid, "Algorithms and software for diffractive optics," Proc. SPIE 3190, Fifth International Topical Meeting on Education and Training in Optics, (8 December 1997); doi: $10.1117 / 12.294364$

Event: Fifth International Topical Meeting on Education and Training in Optics, 1997, Delft, Netherlands 


\title{
Algorithms and software for diffractive optics
}

\author{
Frank Wyrowski ${ }^{a}$, Harald Aagedal ${ }^{a}$, Thomas Beth $^{b}$, and Michael Schmid ${ }^{b}$ \\ ${ }^{a}$ Institut für Angewandte Physik \\ Friedrich-Schiller-Universität Jena, Germany \\ ${ }^{b}$ Institut für Algorithmen und Kognitive Systeme \\ Universität Karlsruhe, Germany
}

\begin{abstract}
The present paper describes the requirements for software for diffractive optics and briefly explains some basic facts and algorithms. Examples from an academically developed software package are given.
\end{abstract}

Keywords: physical optics, diffractive optics, software, algorithms

\section{INTRODUCTION}

The rapid development of computer technology has triggered an enormous increase in the utilization of computers in optics. Academic and commercial software installed on powerful PCs have become an important tool for the numerical analysis of optical phenomena as well as the design of optical components and systems.

Initially, the use of computers in optics was to implement numerical techniques based on geometrical optics for the optimization of refractive systems. As a result, numerous ray-tracing programs are now available and in use in most optical laboratories.

In recent years the development of software for physical optics has become of concern. Wave propagation and the interaction of electromagnetic waves with interfaces are the basic issues in physical optics of linear media. In particular, the optics of laterally microstructured interfaces, i.e. diffractive optics, represents a significant extension of physical optics.

Besides the fundamental research in diffractive optics, the implementation of software in this modern field of optics requires the formulation of efficient algorithms for the analysis and synthesis of laterally microstructured interfaces, i.e. diffractive elements. A basic design theory ${ }^{1}$ allows the formulation of efficient algorithms for the design of diffractive elements in the paraxial domain.

\section{REQUIREMENTS FOR SOFTWARE IN DIFFRACTIVE OPTICS}

To simulate a diffractive system some basic circumstances should be considered.

\subsection{Wave representation}

Electromagnetic waves are solutions of the $3 \mathrm{D}$ wave equation which are fully determined in homogeneous media by a $2 \mathrm{D}$ plane. Thus, in scalar theory electromagnetic waves can be represented by complex-valued $2 \mathrm{D}$ matrices determining amplitude and phase at each point. For the representation of a vectorial wavefront a further field can be specified. The matrix representation requires sampling of the continuous function describing the wavefront. Because of the discrete representation it is important to pay attention to the two contradicting demands of sufficient sampling and memory requirements.

Other author information:

H. A.: Email: aagedal@ira.uka.de; Tel.: ++49-3641-657658;

M. S.: Address: Am Fasanengarten 5, 76128 Karlsruhe; Email: schmidm@ira.uka.de; Tel.: ++49-721-6086291; Fax: ++49-721-696893;

F. W.: Address: Max-Wien-Platz 1, 07743 Jena; Email: wyrowski@uni-jena.de; Tel.: ++49-3641-657640; Fax: ++49-3641-657680; 


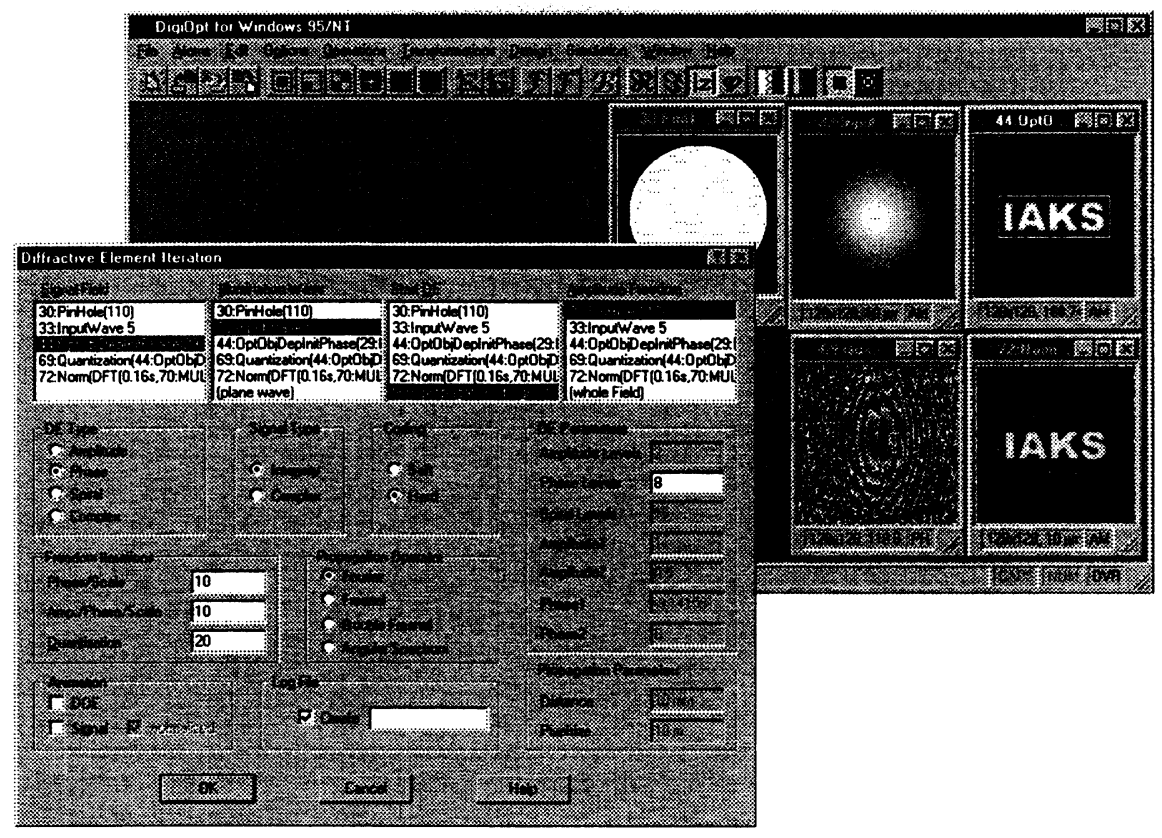

Figure 1. Screen shot showing a dialog for a diffractive element design. In this case an eight-level diffractive phase element was to be computed which transforms a Gaussian wavefront (small window in the middle of the upper row) into the desired far-field intensity distribution showing the letters $I A K S$ (upper right window with the circumscribed region of the signal window). The computed phase element and the resulting wavefront can be seen in the lower row. In addition, the region of amplitude freedom is specified by the window in the left of the upper row.

\subsection{Wave propagation}

One step towards software for diffractive optics is a proper numerical handling of wave propagation operators. ${ }^{2}$ Due to their different numerical and physical properties various operators should be implemented. The difference lies in the physical validity, the numerical correctness of the output and the sampling grid of the resulting wavefront. Their characteristics depend on several parameters such as wavelength, sampling period and propagation distance but also on the spatial properties of the initial wavefront itself. The angular spectrum of plane waves, the Kirchhoff integral, the Rayleigh-Sommerfeld integral, the Fresnel integral and the Debye approximation are the most wellknown wave propagation operators that should be considered included in a software package for physical optics. Moreover, there exist several discrete versions for each of these continuous wave propagation operators with different numerical properties.

Of course, one also has to pay attention to the computation time and memory requirements because wave propagation is the basic and most time consuming part of most design algorithms for diffractive elements. Straightforward implementations of the wave propagation operators normally require $O\left(N^{4}\right)$ operations in order to compute the wave propagation from one surface to another of a field consisting of $N \times N$ sampling points. However, symmetry properties of most discrete wave propagation operators allow to express these in terms of the Fourier transform which again may be computed by the fast Fourier transform (FFT) in only $O\left(N^{2} \log (N)\right)$ steps. Most discrete wave propagation operators can be reduced to one, two or three FFTs with an additional overhead of $O\left(N^{2}\right)$.

Besides the use of wave propagation operators in design algorithms, the simulation of wave propagation greatly contributes to students' understanding of the nature of waves.

\subsection{Effect of microstructured interfaces}

In addition to the behavior of waves in homogeneous media described in the section above it is important to model the interaction between matter and electromagnetic waves. The scalar theory is valid for microstructured interfaces with feature sizes larger than some wavelengths and such optical elements are normally referred to as paraxial diffractive 


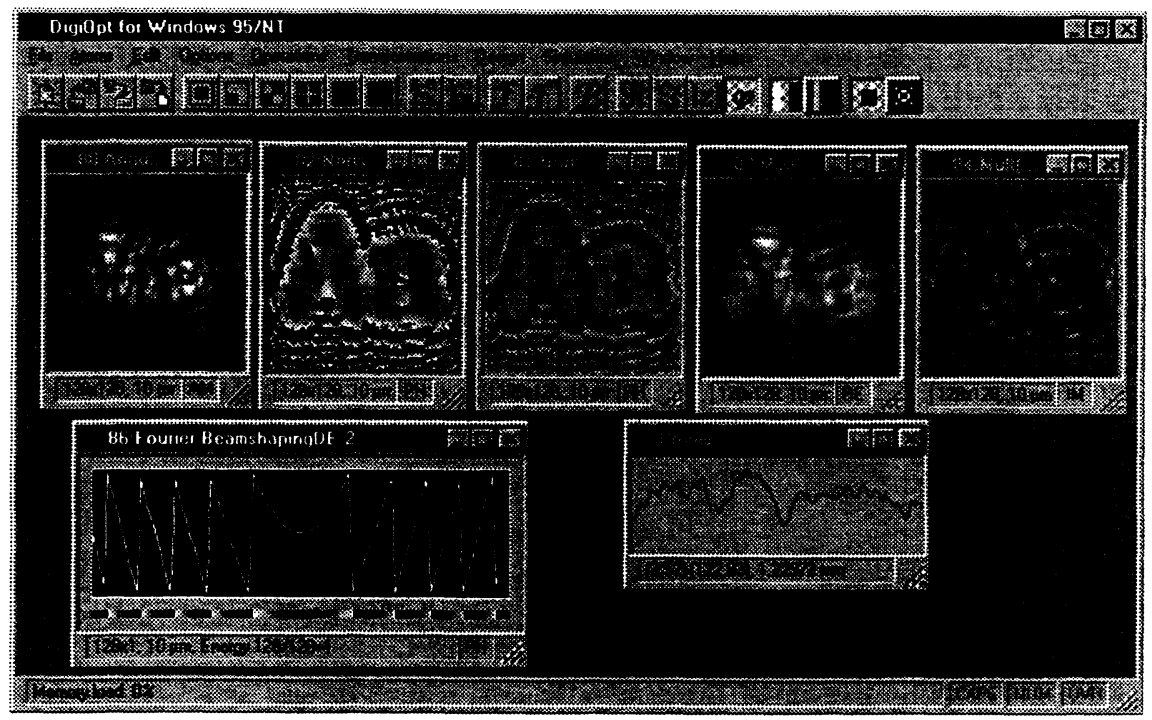

Figure 2. Screenshot of DigiOpt showing different wavefront representations. Each subwindow in the upper row describes a 2D wavefront in different representations (from the left: amplitude, phase in two different color modes, real and imaginary part). In the bottom row a 1D wavefront and a profile of a line intersection of one of the 2D windows can be seen. The statusbar of each window contains context dependent information such as number of pixels, physical pixel size and energy of the wave.

elements. The effect of paraxial diffractive elements on an impinging wavefront can be modelled by the thin-element approximation and the complex-transmission-approach. In this theory the effect of a diffractive element corresponds to a pointwise multiplication of the wavefront with the complex amplitude of the transmission function describing the diffractive element. In this model it is also easy to determine the microstructured interface if the wavefront immediately in front and behind of the element is known, which is important for most design algorithms.

In the case of smaller feature sizes, rigorous wave theory has to be applied to simulate the interaction between the electromagnetic wave and the diffractive element. Some of these methods are known as integral methods, differential methods, the method of moments, BKK, FEM, etc. Unfortunately, a rigorous treatment requires high computational costs. Moreover, the inverse problem of finding an interface from a given incident and diffracted wave is not satisfactorily solved in the non-paraxial region as opposed to the paraxial design problem.

\subsection{Design algorithms}

Modern diffractive optics contains a variety of methods to design diffractive elements with certain optical functions which are mostly based on the paraxial approximation. These include the iterative Fourier transform algorithm (IFTA $)^{3,4}$ also known as the Gerchberg-Saxton algorithm, direct binary search (DBS), ${ }^{5}$ simulated annealing (SA), ${ }^{6}$ genetic algorithms (GA), different gradient methods, Dammann grating methods, ${ }^{7}$ geometrical beam shaping methods, ${ }^{8,9}$ etc. Of course, some of these should be contained in a software package for the educational and scientific use in diffractive optics. To demonstrate the complexity of some of these algorithms a screenshot of the IFTA dialog of the academically developed software package DigiOpt ${ }^{10,11}$ is shown in figure 1.

\subsection{User-friendliness}

A software package for diffractive optics should present itself with a user-friendly interface suitable for the untrained as well as the more experienced user. The position in the trade-off between user-friendliness and complexity should be carefully chosen. An example of a screenshot of a possible implementation is given in figure 2. Important points for the user-friendliness are in particular

- visualization of optical elements and wavefronts in different representations 


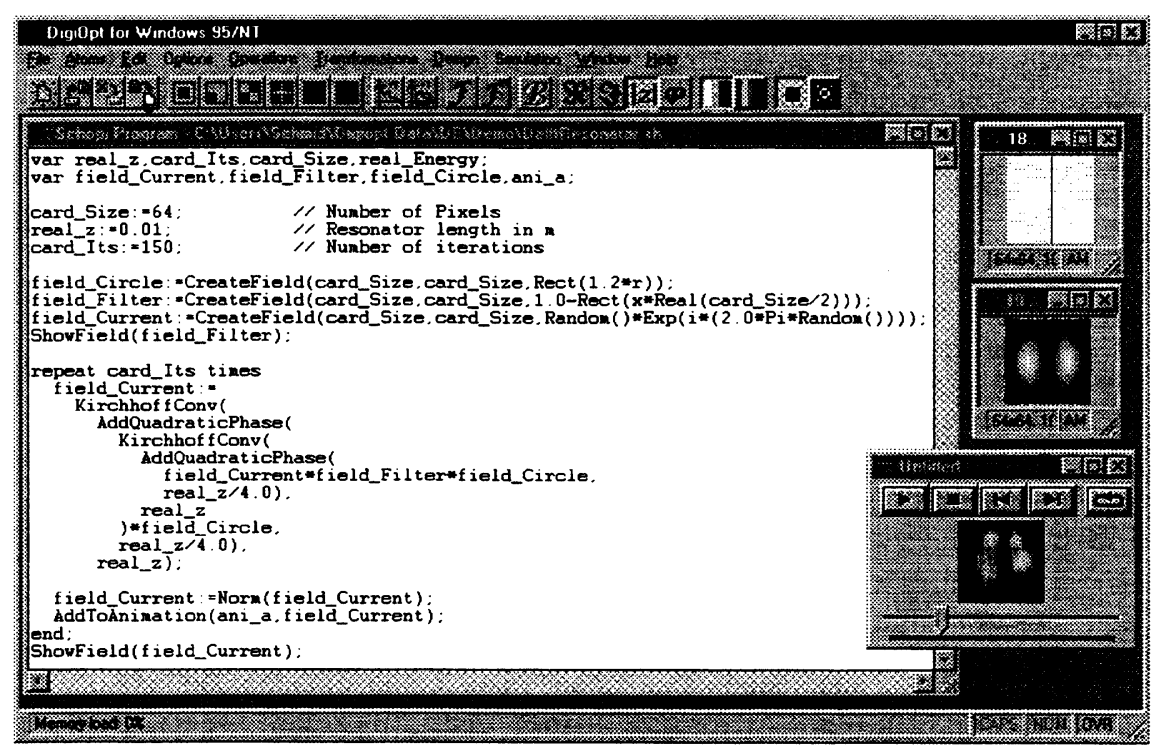

Figure 3. The text window shows a program performing a Fox-Li algorithm ${ }^{12}$ which simulates an optical resonator with an internal optical element to induce higher modes (in this case a TEM $_{01}$ mode). The upper window shows the function of the internal optical mode filter. Beneath the output of the resonator after 150 roundtrips can be seen and in the bottom window an animation of the mode development is depicted.

- easy access to basic functions of the software

- implementation of standard image processing and file handling features

- interfaces and matching file formats to standard software (graphics, mathematics, fabrication utilities, ...)

- online help system and documentation

- the user should be warned if he is about to perform physically illegal operations or encounters numerical problems. Hints on how to overcome these problems should be given.

\subsection{Flexibility}

A strength of good software is the ability to enhance the built-in features. Thus the software should offer the possibility for the user to easily develop and change self-written or existing algorithms. This can be customized by a higher-level language designed for diffractive optics purposes. With such a programming language the student has the possibility to understand well-known algorithms by implementing these on his own. For the scientific user high-level programming offers the possibility to develop new algorithms quickly and to experiment with these in an easy way. Thus rapid prototyping of optical systems is possible.

As an example of the power of high-level programming figure 3 shows a screenshot of DigiOpt with a short program in the integrated programming language simulating an optical resonator. It can be seen that only a few lines of code are necessary to implement relatively complex algorithms if the language is appropriate.

\section{CONCLUSION}

In conclusion, a software package for educational and scientific use in diffractive optics should fulfil the following criteria:

1. user-friendliness 
2. appropriate wavefront modeling

3. implementation of various wave propagation operators

4. appropriate modelling of the effect of microstructured interfaces

5. flexibility

\section{REFERENCES}

1. F. Wyrowski and O. Bryngdahl, "Digital holography as part of diffractive optics," Rep. Prog. Phys. 54, pp. 1481$1571,1991$.

2. H. Aagedal, S. Egner, M. Schmid, and T. Beth, "Important aspects of the numerical implementation of wave propagation between parallel planes demonstrated with the CAD system DigiOpt," in Diffractive Optics. EOS Topical Meeting Digest Series, vol. 12, p. 162, EOS-European Optical Society, 1997.

3. R. W. Gerchberg and W. O. Saxton, "A practical algorithm for the determination of phase from image and diffraction plane pictures," Optik 35, pp. 237-246, 1972.

4. F. Wyrowski and O. Bryngdahl, "Iterative fourier-transform algorithm applied to computer holography," $J$. Opt. Soc. Am. A 5, pp. 1058-1065, July 1988.

5. M. A. Seldowitz, J. P. Allebach, and D. W. Sweeney, "Synthesis of digital holograms by direct binary search," Appl. Optics 28, pp. 2788-2798, 1987.

6. J. Turunen, A. Vasara, and J. Westerholm, "Kinoform phase relief synthesis: a stochastic method," Opt. Eng. 28, pp. 1162-1167, Nov. 1989.

7. H. Dammann and E. Klotz, "Coherent optical generation and inspection of two-dimensional periodic structures," Optica Acta 24(4), pp. 505-515, 1977.

8. C.-Y. Han, Y. Ishii, and K. Murata, "Reshaping collimated laser beams with gaussian profile to uniform profiles," Appl. Optics 22, pp. 3644-3647, Nov. 1983.

9. H. Aagedal, M. Schmid, S. Egner, J. Müller-Quade, T. Beth, and F. Wyrowski, "Analytical beam shaping with application to laser diode arrays," J. Opt. Soc. Am. A 14, pp. 1549-1553, July 1997.

10. H. Aagedal, T. Beth, H. Schwarzer, and S. Teiwes, "Modern concepts for computer-aided design in diffractive optics," in OSA Proceedings of the International Optical Design Conference, G. W. Forbes, ed., vol. 22, pp. 257260, Optical Society of America, Washington DC, 1994.

11. H. Aagedal, T. Beth, H. Schwarzer, and S. Teiwes, "Design of paraxial diffractive elements with the computeraided design (CAD) system DigiOpt," in Diffractive and Holographic Optics Technology II, I. Cindrich and S. H. Lee, eds., vol. 2404, pp. 50-58, SPIE-The International Society for Optical Engineering, 1995.

12. A. G. Fox and T. Li, "Resonant modes in a maser interferometer," Bell. Syst. Tech. J. 40, pp. 453-488, Jan. 1961. 